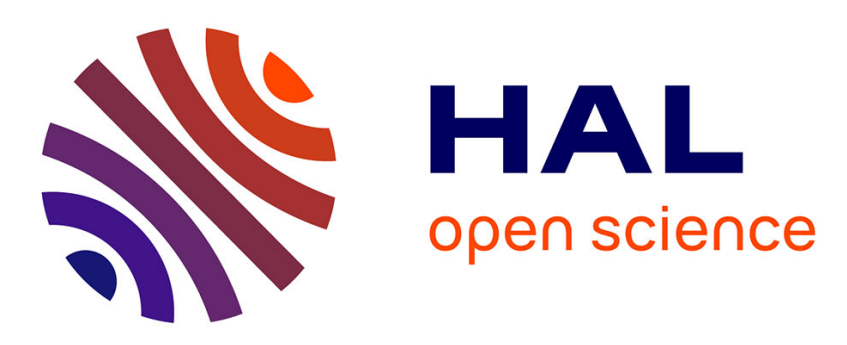

\title{
Modeling ASR Ambiguity for Neural Dialogue State Tracking
}

Vaishali Pal, Fabien Guillot, Manish Shrivastava, Jean-Michel Renders, Laurent Besacier

\section{- To cite this version:}

Vaishali Pal, Fabien Guillot, Manish Shrivastava, Jean-Michel Renders, Laurent Besacier. Modeling ASR Ambiguity for Neural Dialogue State Tracking. Interspeech 2020, Oct 2020, Shangai (Virtual Conf), China. hal-02962177

\section{HAL Id: hal-02962177 \\ https://hal.science/hal-02962177}

Submitted on 9 Oct 2020

HAL is a multi-disciplinary open access archive for the deposit and dissemination of scientific research documents, whether they are published or not. The documents may come from teaching and research institutions in France or abroad, or from public or private research centers.
L'archive ouverte pluridisciplinaire HAL, est destinée au dépôt et à la diffusion de documents scientifiques de niveau recherche, publiés ou non, émanant des établissements d'enseignement et de recherche français ou étrangers, des laboratoires publics ou privés. 


\title{
Modeling ASR Ambiguity for Neural Dialogue State Tracking
}

\author{
Vaishali Pal $^{1,3}$, Fabien Guillot ${ }^{1}$, Manish Shrivastava $^{3}$, Jean-Michel Renders ${ }^{1}$, Laurent Besacier ${ }^{1,2}$ \\ ${ }^{1}$ Naver Labs Europe \\ ${ }^{2}$ LIG - Université Grenoble Alpes, France \\ ${ }^{3}$ International Institute of Information Technology, Hyderabad, India \\ vaishali.paleresearch.iiit.ac.in, jean-michel.renders@naverlabs.com, \\ laurent.besacier@univ-grenoble-alpes.fr
}

\begin{abstract}
Spoken dialogue systems typically use one or several (top-N) ASR sequence(s) for inferring the semantic meaning and tracking the state of the dialogue. However, ASR graphs, such as confusion networks (confnets), provide a compact representation of a richer hypothesis space than a top-N ASR list. In this paper, we study the benefits of using confusion networks with a neural dialogue state tracker (DST). We encode the 2dimensional confnet into a 1-dimensional sequence of embeddings using a confusion network encoder which can be used with any DST system. Our confnet encoder is plugged into the 'Global-locally Self-Attentive Dialogue State Tacker' (GLAD) model for DST and obtains significant improvements in both accuracy and inference time compared to using top-N ASR hypotheses.
\end{abstract}

Index Terms: speech recognition, dialog state tracking, confusion network, attention model

\section{Introduction}

Spoken task-oriented dialogue systems guide the user to complete a certain task through speech interaction. While such speech systems generally include an explicit automatic speech recognition (ASR) module (cascade approach), they now tend to be replaced by end-to-end approaches where the systems take speech as input and directly produce a decision from it. Examples include end-to-end architectures for spoken language understanding (SLU) proposed recently [1, 2, 3, 4]. However, those end-to-end models currently lead to equivalent but no better performance compared to cascade approaches based on ASR (see for instance [1, 2]). Besides, in some specific use cases, it may be preferable to deploy modular systems (instead of a monolithic one) for which only one component (ASR, SLU, dialog state tracker) can be modified at a time.

This article is positioned in this latter context and studies how to take better account of ASR ambiguity in voice-based dialog state tracking systems. Most recent work on spoken dialogue systems uses one or several (top-N) ASR sequence(s) to track the dialogue state and infer user needs. However, ASR lattices provide a richer hypothesis space than the top- $\mathrm{N}$ hypotheses. More precisely, we revisit the use of word confusion networks (simply denoted as confnets) [5], derived from ASR lattices, as a compact and efficient representation of ASR output. To encode such graphical representations with existing stateof-the-art dialogue state trackers (DST), we introduce a generic neural confusion network encoder (see Figure 1) which can be used as a plug-in to any dialogue state tracker and achieves better results than using a list of top-N ASR hypotheses.

Our research contributions are the following:
- we introduce a system which encodes the confusion network into a representation that can be used as a plug-in to any state-of-the-art dialogue state tracker,

- we propose and experiment several variants to encode ASR confnets,

- we introduce mechanisms to leverage both ASR confusion network and true transcripts while training the DST system,

- we explore DST using a richer hypothesis space in the form of a confusion network and study whether it leads to better performance / computation trade-off compared to using a list of top-N ASR hypotheses.

\section{Related Work}

Dialog state tracking. Recent pieces of work on dialogue state trackers $[6,7,8]$ infer the state of the dialogue from conversational history and current user utterance. These systems assume a text-based user utterance and accumulate the user goal across multiple user turns in the dialogue. [6] generalizes on rare slotvalue pairs by using global modules which share parameters and local modules to learn slot specific feature representations. [7] achieves state-of-the-art performance on DSTC-2 dataset [9] with a universal state tracker which generates fixed-length representation for each slot and compares the distance between the representation and value vectors to make predictions. [10] predicts dialogue states from utterances and schema graphs containing slot relations in edges to achieve state-of-the-art result of MultiWoz 2.0 [11] and MultiWoz 2.1 datasets [12].

Using ASR graphs with neural models. Word lattices from ASR were used by [13] for intent classification in SLU with RNNs. Inspired from [13], [14] proposed to use word confusion networks for DST. However, there are several differences with what we propose in our paper: (1) they only use average pooling to aggregate the hidden GRU states corresponding to the alternative word hypotheses whereas we introduce several variants to pool alternative words (see section 3), (2) our word confusion network encoder can be plugged into any neural architecture for dialogue state tracking, as it basically amounts to have a first layer that transforms a 2D-data structure (confnets) into a 1D-sequence of embeddings, while [14] keeps the 2Ddata structure in the hidden layers and, consequently, is limited to simple RNNs such as GRU and (bi-)LSTM, and (3) they experiment with a simpler RNN-based dialog state tracker while we plug our confnet encoder into the more efficient 'Globallocally Self-Attentive Dialogue State Tacker' (GLAD) model of [6]. Finally, our confnet encoder is most similar to [15] but they used ASR confnets for classification of user intent, questiontype and named-entities while we apply our encoder to a DST task (we also propose several variants over [15]). 


\section{Word Confusion Network for DST}

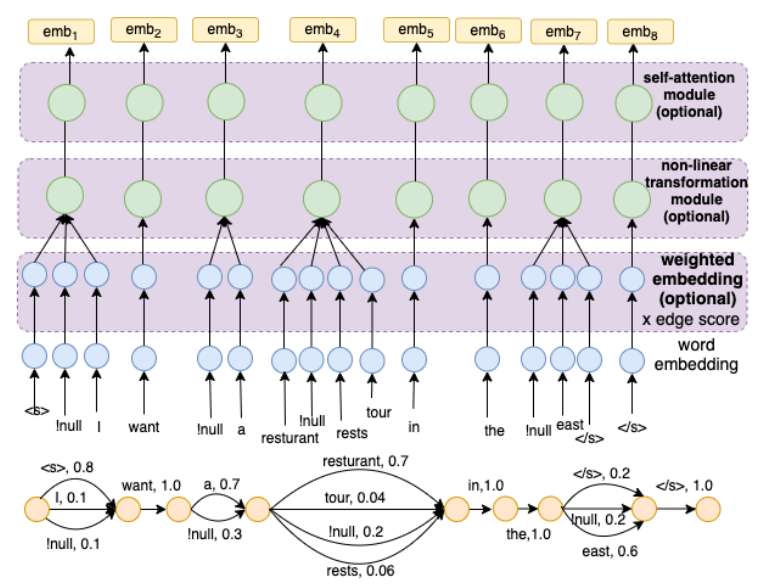

Figure 1: Word Confusion Network Encoder Variations

\subsection{Confusion Network Encoder}

Inspired from [15], we use a word confusion network encoder to transform the graph to a representation space which can be used with any dialogue state tracker. The multiple aligned ASR hypotheses, represented as parallel arcs at each position of the confusion network, are treated as a set of weighted arcs. More formally, a confnet $C$ is a sequence of parallel weighted arcs, noted as $C=\left[\left(<w_{1}^{1}, \pi_{1}^{1}>,<w_{1}^{2}, \pi_{1}^{2}>, \ldots,<w_{1}^{n_{1}}, \pi_{1}^{n_{1}}>\right.\right.$ )$\left., \ldots,\left(<w_{m}^{1}, \pi_{m}^{1}>,<w_{m}^{2}, \pi_{m}^{2}>, \ldots,<w_{m}^{n_{m}}, \pi_{m}^{n_{m}}>\right)\right]$, where $w_{t}^{j}$ is the $j^{t h}$ arc (token) at time/position $t$, and $\pi_{t}^{j}$ its associated confidence weight. We propose several variants to formulate the embedding representation for a set $C_{t}$ of parallel arcs at position $t$ in the confnet $C$.

The simplest method to encode the confusion network is as a sequence of weighted-sums of the word embeddings weighed with the ASR confidence scores:

$$
\begin{aligned}
p_{t}^{i} & =\pi_{t}^{i} \text { Embedding }\left(w_{t}^{i}\right) \\
e_{v 1}\left(C_{t}\right) & =\sum_{i} p_{t}^{i}
\end{aligned}
$$

In increasing complexity, the second variant to encode the parallel arcs is to apply a weighted sum of non-linear transformations over word embeddings:

$$
\begin{aligned}
r_{t}^{i} & =\pi_{t}^{i} \tanh \left(W_{1} \text { Embedding }\left(w_{t}^{i}\right)\right) \\
e_{v 2}\left(C_{t}\right) & =\sum_{i} r_{t}^{i}
\end{aligned}
$$

The third variation is to formulate the encoding with a selfattention mechanism similar to that described in [15]. In this case, $\pi_{t}^{i}$ (ASR) weights can be ignored as the model will use self-attention to weight the parallel arcs. The self-attention weights $\alpha_{t}^{i}$ are learnt during training:

$$
\begin{aligned}
q_{t}^{i} & =\tanh \left(W_{1} \text { Embedding }\left(w_{t}^{i}\right)\right) \\
\alpha_{t}^{i} & =\frac{\exp \left(w_{2}^{T} q_{t}^{i}\right)}{\sum_{j} \exp \left(w_{2}^{T} q_{t}^{j}\right)} \\
e_{v 3}\left(C_{t}\right) & =\sum_{i} \alpha_{t}^{i} q_{t}^{i}
\end{aligned}
$$

Embedding $\left(w_{t}^{i}\right)$ is the embedding representation of word $w_{t}^{i}$. The final variation is to use the self-attention mechanism exactly as described in [15]. The ASR weights $\pi_{t}^{i}$ are used as an additional feature to weigh the word embeddings of each parallel arc:

$$
\begin{aligned}
\bar{q}_{t}^{i} & =\tanh \left(W_{1} p_{t}^{i}\right) \\
\bar{\alpha}_{t}^{i} & =\frac{\exp \left(w_{2}^{T} \bar{q}_{t}^{i}\right)}{\sum_{j} \exp \left(w_{2}^{T} q_{t}^{\bar{j}}\right)} \\
e_{v 4}\left(C_{t}\right) & =\sum_{i} \bar{\alpha}_{t}^{i} \overline{q_{t}^{i}}
\end{aligned}
$$

$e_{v x}$ denotes the 4 variations of the standard trainable embedding layer for word/token $w_{t}^{i}$; the matrix $W_{1}$ and the vector $w_{2}$ are trainable parameters of our model. Note that the training of these parameters is done jointly with the main task (see next subsection).

\subsection{Dialogue State Tracking with Confnet}

The dialogue state is a representation of the user goal at any time in the dialogue. A dialogue state tracker (DST) accumulates evidence as the dialogue progresses at each user turn and updates the state to reflect the changing user goals. The usergoal is captured by the tracker as a distribution of slot-value pairs. Each user utterance can be either in textual or spoken form. Conventionally, DST uses top-N list of ASR hypotheses of the spoken user utterances to track the user needs. However, graph based representation such as ASR lattices and confusion network provides a richer hypothesis space in compact form.

Our confusion network encoder can be used as a plug-in to any state-of-the-art DST system. We have used the 'Globallocally Self-Attentive Dialogue State Tacker' (GLAD) model [6] with our confusion network encoder. GLAD addresses the issue of rare slot-value pairs which were not explicitly handled by previous DST models. The GLAD encoder module is a global-local self-attentive encoder which separately encodes the transcript/ASR hypothesis, system actions from previous turns and slot-value under consideration. We extend GLAD by replacing the user utterance representation, namely a sequence of trainable token embeddings, by the confnet embedding sequence. Remind that a confnet is also encoded as a 1 dimensional sequence of embeddings that corresponds to each time/position in the confnet. This enables GLAD architecture to use graph-based inputs instead of (or even in addition to) token sequence inputs.

\section{Model Training Strategies}

At training time, both clean transcript and ASR graph are available. It is therefore tempting to use these two pieces of information to facilitate model training while trying to make it robust to ASR errors in the meantime. We propose two radically different strategies to take into account clean transcript and ASR graph at training time.

\subsection{Data Augmentation}

The confusion network contains noisy hypotheses with lower ASR confidence scores which makes training hard. Augmenting the confnet dataset with the clean transcript should help the system to converge faster and better. We encode transcript in the form of a confnet with a single arc between nodes. At training time we merge both noisy ASR and clean (single arc) confnet datasets. Consequently, we use each dialog twice at training. 


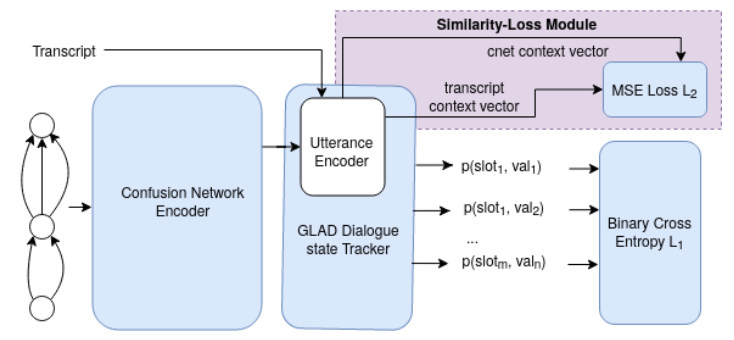

Figure 2: Confusion Network Encoder used with DST system (GLAD). Similarity-loss module can be used for augmenting the transcript data to jointly-train the system.

\subsection{Adding a Similarity Loss to Train Confnet Embed- dings: Jointly-Trained Model}

We want the confnet representation to be very close to the one of the clean transcript in the embedding space. To enforce this, we add a similarity loss to the loss corresponding to our main task (binary classification for each $<$ slot,value $>$ pair) - see figure 2 .

Binary-cross-entropy is used as classification loss, denoted as $L_{1}$, and squared euclidean distance between clean and noisy transcripts is used as similarity loss $\left(L_{2}\right)$. The binary crossentropy loss can be modeled as shown in equation 11 where $p\left(\right.$ slot $\left._{m}, v \mathrm{l}_{n}\right)$ represents the prediction for the slot $m$ and value $n$ and $y$ is the binary ground-truth. Let Embedding ${ }_{C N}$ be the transformation realised by the confnet encoder and Embedding $_{T}$ be the standard embedding layer on word tokens; $f$ be the transformation function of the GLAD encoder that takes as input the sequence of one dimensional embeddings (either standard word token embeddings or confnet embeddings) and outputs a global-local context vector. The final loss, $L$, of the model is defined as a linear combination of the cross-entropy loss, $L_{1}$, and the similarity loss, $L_{2}$ as shown in equation 11. We choose a $\lambda$ value of 0.5 for our experiments.

$$
\begin{gathered}
L=L_{1}+\lambda L_{2} \\
L_{1}=-(y \log (P)+(1-y) \log (1-P)) \\
P=p\left(\text { slot }_{m}, \text { val }_{n}\right) \\
L_{2}=\| f\left(\text { Embedding }_{T}(\text { transcript })\right) \\
-f\left(\text { Embedding }_{C N}(\text { confnet })\right) \|_{2}^{2}
\end{gathered}
$$

Note that, while $L_{2}$ alone has a trivial solution (namely, projecting everything to a null vector), the combination with the loss associated to the primary task renders this trivial solution non-optimal. The model trained jointly using the similarity loss $L_{2}$ is called JCnet.

\section{DSTC-2 Dataset}

We evaluate our system on the standard Dialogue State Tracking Challenge 2 (DSTC-2) dataset [9]. ${ }^{1}$ DSTC is a research challenge focused on improving the state-of-the art in tracking the state of spoken dialog systems. DSTC 2 contains dialogue in the restaurant information domain where the states may change through the dialogue. There are 1612, 506 and 1117 dialogues in the training, development and test sets respectively. The dialog state is captured by informable slots (slots which the user can provide a value for, to use as a constraint on their search)

\footnotetext{
${ }^{1}$ http://camdial.org/ mh521/dstc/
}

and non informable (unconstrained) slots. All the slots in the dataset are requestable as the user can request the value of any slot. For example, for a given restaurant, the user can request the value of the phone number or price-range slot. At each turn of the dialogue, the user's goal may change, new information maybe requested or provided to the system by the user. Thus, a turn is represented as a dialogue state comprising of the triplet (user-goal, turn-request, turn-inform). DSTC-2 provides representation of the user speech utterance in the form of top-10 ASR hypotheses and word confusion networks.

We followed a similar pre-processing pipeline of the confusion networks as mentioned in [14], i.e, we removed the interjections (um, ah, etc) and pruned arcs with an ASR confidence score less than 0.001 to reduce the size of the network, thus increasing efficiency without compromising the accuracy of models. To obtain a fair and consistent performance comparison between models based on ASR-top- $\mathrm{N}$ hypotheses and on confusion network, the top-N list of ASR hypotheses was chosen as the $\mathrm{N}$ best paths extracted from the confusion network.

\section{Experiments and Results}

Our baseline is the model trained on augmented dataset composed of ASR-N hypotheses and transcripts, where the final prediction is the weighted sum of prediction probabilities from each ASR hypothesis (Augmented ASR-N, see table 1). To demonstrate that a richer hypothesis space (confusion network) helps in improving accuracy, we trained 3 separate models on confusion networks with non-augmented (Non-augmented Cnet- $N$ ), augmented (Augmented Cnet- $N$ ) dataset and jointlytrained model $($ JCnet $-N)$. In these models, we restricted the number of arcs per token by keeping only the ones with the top- $N$ weights $(N \in[5,9])$. The augmented dataset is composed of transcripts modeled as a graph with one arc between nodes, and ASR confusion networks. In addition to the above data augmentation techniques, we also evaluate the impact of using a similarity loss to train the confnet embeddings as introduced in section $4.2($ JCnet- $N)$. We use a learning rate of 0.01 , a batch size of 50, a dropout of 0.2 and a $\lambda$ value of 0.5 to train our models. We concatenate pre-trained word embeddings (GloVe) [16] and Kazuma character embeddings [17] to encode words. The embedding layer is frozen and not updated during training.

Table 1: Scores for baseline model trained on augmented ASR$N$ list of hypothesis. Each cell contains the mean accuracy $\mu(0-$ $1)$ and standard error $\sigma(0-1)$ in the format $\mu(\sigma)$ for 4 runs of each setting

\begin{tabular}{|c|c|c|c|}
\hline List-Size & Joint-Goal & Turn-Inform & Turn-Request \\
\hline ASR-1 & $0.6846(.0017)$ & $0.8326(.0012)$ & $0.9668(.0003)$ \\
ASR-5 & $0.6980(.0075)$ & $0.8375(.0050)$ & $0.9680(.0010)$ \\
ASR-9 & $0.6942(.0077)$ & $0.8395(.0055)$ & $0.9680(.0073)$ \\
\hline
\end{tabular}

Table 2: Scores for Jointly-trained (Similarity Loss) model on augmented confnet dataset. Each cell contains the mean accuracy $\mu(0-1)$ and standard error $\sigma(0-1)$ in the format $\mu(\sigma)$ for 4 runs of each setting

\begin{tabular}{|c|c|c|c|}
\hline Arc-Size & Joint-Goal & Turn-Inform & Turn-Request \\
\hline JCnet-1 & $0.6883(.0027)$ & $0.8361(.0021)$ & $0.9672(.0001)$ \\
JCnet-5 & $0.7088(.0021)$ & $0.8403(.0015)$ & $0.9773(.0003)$ \\
JCnet-9 & $0.7063(.0049)$ & $0.8461(.0048)$ & $0.9700(.0005)$ \\
\hline
\end{tabular}




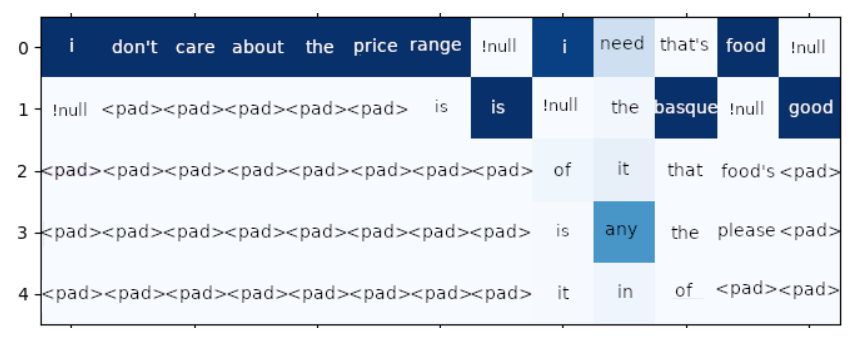

Figure 3: Attention Weights of the confusion network encoder (variant $e_{v_{4}}$ ) for the confnet with transcript ' $i$ don't care about the price range i need basque food'. Dark colors represent high values and light colors low values. The columns represent subsequent parallel arcs. The parallel arcs are sorted from highest to lowest scored hypothesis. The 1st row is the best-pass through the network. The highest attention-weights are for the words ' $i$ don't care about the price range is $i$ any basque food good'

Table 3: Results on confusion network model encoding variants with different numbers of parallel arcs (5 or 9). With or w/o confnet augmentation. Each cell contains the mean accuracy $\mu(0-1)$ and standard error $\sigma(0-1)$ of the 3 metrics on 10 runs of each setting in the format $\mu(\sigma)$

\begin{tabular}{|c|c|c|c|c|c|c|}
\hline Accuracy $[\mu(\sigma)]$ & Joint-Goal & Turn-Inform & Turn-Request & Joint-Goal & Turn-Inform & Turn-Request \\
\hline Parallel Arcs: 5 & \multicolumn{3}{|c|}{ Confnet Encoding Variation 1: $e_{v 1}=\sum_{i} p_{t}^{i}$} & \multicolumn{3}{|c|}{ Confnet Encoding Variation 2: $e_{v 2}=\sum_{i} r_{t}^{i}$} \\
\hline Non-aug CNET-N & $0.7019(0.0026)$ & $0.8350(0.003)$ & $0.9690(0.0004)$ & $0.7008(0.0027)$ & $0.8389(0.002)$ & $0.9677(0.0003)$ \\
\hline \multirow[t]{2}{*}{ Aug CNET-N } & $0.7121(0.0019)$ & $0.8470(0.0011)$ & $0.9688(0.0004)$ & $0.7115(0.0019)$ & $0.8465(0.0011)$ & $0.9686(0.0005)$ \\
\hline & \multicolumn{3}{|c|}{ Confnet Encoding Variation 3: $e_{v 3}=\sum_{i} \alpha_{t}^{i} q_{t}^{i}$} & \multicolumn{3}{|c|}{ Confnet Encoding Variation 4: $e_{v 4} \sum_{i} \overline{\alpha_{t}^{i}} \overline{\overline{q_{t}^{i}}}$} \\
\hline Non-aug CNET-N & $0.6912(0.0057)$ & $0.8302(0.0037)$ & $0.9645(0.0005)$ & $0.6975(0.0016)$ & $0.8361(0.0013)$ & $0.9686(0.0004)$ \\
\hline Aug CNET-N & $0.6925(0.0032)$ & $0.8372(0.0008)$ & $0.9650(0.0006)$ & $0.7056(0.0023)$ & $0.8413(0.0015)$ & $0.9689(0.0007)$ \\
\hline Parallel Arcs: 9 & \multicolumn{3}{|c|}{ Confnet Encoding Variation 1: $e_{v 1}=\sum_{i} p_{t}^{i}$} & \multicolumn{3}{|c|}{ Confnet Encoding Variation 2: $e_{v 2}=\sum_{i} r_{t}^{i}$} \\
\hline Non-aug CNET-N & $0.7044(0.0020)$ & $0.8395(0.0009)$ & $0.9693(0.0003)$ & $0.6997(0.0043)$ & $0.8349(0.0038)$ & $0.9684(0.0003)$ \\
\hline \multirow[t]{2}{*}{ Aug CNET-N } & $0.7094(0.0017)$ & $0.8446(0.0012)$ & $0.9694(0.0004)$ & $0.7071(0.0095)$ & $0.8413(0.0057)$ & $0.9680(0.0004)$ \\
\hline & \multicolumn{3}{|c|}{ Confnet Encoding Variation 3: $e_{v 3}=\sum_{i} \alpha_{t}^{i} q_{t}^{i}$} & \multicolumn{3}{|c|}{ Confnet Encoding Variation 4: $e_{v 4}=\sum_{i} \overline{\alpha_{t}^{i}} \overline{q_{t}^{i}}$} \\
\hline Non-aug CNET-N & $0.6938(0.0033)$ & $0.8325(0.0022)$ & $0.9650(0.0003)$ & $0.6969(0.0023)$ & $0.8382(0.0011)$ & $0.9680(0.0006)$ \\
\hline Aug CNET-N & $0.6930(0.0021)$ & $0.8367(0.0007)$ & $0.9648(0.0006)$ & $0.7045(0.0023)$ & $0.8416(0.0011)$ & $0.9681(0.0008)$ \\
\hline
\end{tabular}

We train each experimental setting multiple times with different seeds and report the mean accuracy $(\mu)$ and standard error $(\sigma)$ of the joint-goal, turn-inform and turn-request for the ASR$\mathrm{N}$ models in table 1, JCnet- $N$ models in table 2 and the variants of confusion network encoder models in table 3 . Our comments will mostly focus on the joint-goal accuracy metric which is the most important. We use 10 runs to calculate $\mu$ and $\sigma$ for Non-augmented Cnet- $N$ and Augmented Cnet- $N$ models and 4 runs to calculate $\mu$ and $\sigma$ for Augmented ASR-N and JCnet- $N$ models. As illustrated in table 3 , the joint-goal accuracy of the models trained on augmented dataset (leveraging clean transcripts at training time) performs better than those trained on non-augmented one. These models also outperform the ASR$\mathrm{N}$ baseline. The best variants seem to be $e_{v_{1}}$ and $e_{v_{2}}$ which do not use attention, both outperforming $e_{v_{4}}[15]$. However, attention models like $e_{v_{4}}$ interestingly learn to assign higher weights to relevant words with lower ASR score over the top1 hypothesis as shown in the figure 3. Content words, such as 'basque' which aids in classifier discriminability, is chosen by the attention-weights over function words such as 'that's' inspite of the later being the top hypothesis. Furthermore, the performance of jointly-trained (similarity loss) confnet models in table 2 perform similar to the data augmented confnet models in table 3. Augmented confusion network with parallel arc size 5 (variant $e_{v_{2}}$ ) outperforms jointly-trained model by a margin of $1 \%$ in joint-goal accuracy whereas both models have similar performance with parallel arc size 9 .

The confusion network models lead to significant inference time gains over those trained on the list of ASR hypothesis. ASR-N models aggregate the predictions over each hypothesis to formulate the final prediction, resulting in a time complexity of $O(N M)$ where $N$ is the number of ASR hypothesis and $M$ is the size of the neural network. The confusion network models eliminate the additional time complexity introduced by the ASR hypothesis size without compromising on the rich hypothesis space. Thus, all variations of the confusion network models have an inference time complexity of $O(M)$. Inference time for all the varying confnet models (augmented, non-augmented, and joint goal) is on an average $0.82 \mathrm{sec}$ per batch over a batch-size of 50. The average inference per batch for the ASR-N models progressively increases from $0.57 \mathrm{sec}$ for ASR-1 to a maximum of $7 \mathrm{sec}$ for ASR-10.

\section{Conclusion}

In this paper, we have demonstrated that exploiting the rich hypothesis space of a confusion network instead of being limited to the top-N ASR hypotheses for DST leads to performance gain in time and accuracy. The time gain is significant if we want to incorporate a larger set of alternative ASR hypotheses. Moreover, we explore variations of designing the initial embedding layer transforming the confnet as a one-dimensional sequence of position-wise embeddings which can be plugged into numerous state-of-the-art text-based DST systems. 


\section{References}

[1] D. Serdyuk, Y. Wang, C. Fuegen, A. Kumar, B. Liu, and Y. Bengio, "Towards end-to-end spoken language understanding," CoRR, vol. abs/1802.08395, 2018. [Online]. Available: http://arxiv.org/abs/1802.08395

[2] A. Caubrière, N. A. Tomashenko, A. Laurent, E. Morin, N. Camelin, and Y. Estève, "Curriculum-based transfer learning for an effective end-to-end spoken language understanding and domain portability," CoRR, vol. abs/1906.07601, 2019. [Online]. Available: http://arxiv.org/abs/1906.07601

[3] L. Lugosch, M. Ravanelli, P. Ignoto, V. S. Tomar, and Y. Bengio, "Speech model pre-training for end-to-end spoken language understanding," CoRR, vol. abs/1904.03670, 2019. [Online]. Available: https://arxiv.org/abs/1904.03670

[4] M. Dinarelli, N. Kapoor, B. Jabaian, and L. Besacier, "A data efficient end-to-end spoken language understanding architecture," 2020.

[5] L. Mangu, E. Brill, and A. Stolcke, "Finding consensus in speech recognition: word error minimization and other applications of confusion networks," Computer Speech \& Language, vol. 14, no. 4, pp. 373-400, 2000. [Online]. Available: https://doi.org/10.1006/csla.2000.0152

[6] V. Zhong, C. Xiong, and R. Socher, "Global-locally self-attentive encoder for dialogue state tracking," in Proceedings of the 56th Annual Meeting of the Association for Computational Linguistics (Volume 1: Long Papers). Melbourne, Australia: Association for Computational Linguistics, Jul. 2018, pp. 1458-1467.

[7] L. Ren, K. Xie, L. Chen, and K. Yu, "Towards universal dialogue state tracking," CoRR, vol. abs/1810.09587, 2018. [Online]. Available: http://arxiv.org/abs/1810.09587

[8] N. Mrksic, D. Ó. Séaghdha, T. Wen, B. Thomson, and S. J Young, "Neural belief tracker: Data-driven dialogue state tracking," CoRR, vol. abs/1606.03777, 2016. [Online]. Available: http://arxiv.org/abs/1606.03777

[9] M. Henderson, B. Thomson, and J. D. Williams, "The second dialog state tracking challenge," in Proceedings of the 15th Annual Meeting of the Special Interest Group on Discourse and Dialogue (SIGDIAL). Philadelphia, PA, U.S.A.: Association for Computational Linguistics, Jun. 2014, pp. 263-272. [Online]. Available: https://www.aclweb.org/anthology/W14-4337

[10] L. Chen, B. Lv, C. Wang, S. Zhu, B. Tan, and K. Yu, "Schema-guided multi-domain dialogue state tracking with graph attention neural networks," in AAAI 2020, 2020. [Online] Available: https://www.aaai.org/Papers/AAAI/2020GB/AAAIChenL.10030.pdf

[11] P. Budzianowski, T.-H. Wen, B.-H. Tseng, I. Casanueva, S. Ultes, O. Ramadan, and M. Gašić, "MultiWOZ large-scale multi-domain wizard-of-Oz dataset for task-oriented dialogue modelling," in Proceedings of the 2018 Conference on Empirical Methods in Natural Language Processing. Brussels, Belgium: Association for Computational Linguistics, Oct.-Nov. 2018, pp. 5016-5026. [Online]. Available: https://www.aclweb.org/anthology/D18-1547

[12] M. Eric, R. Goel, S. Paul, A. Sethi, S. Agarwal, S. Gao, and D. Hakkani-Tür, "Multiwoz 2.1: Multi-domain dialogue state corrections and state tracking baselines," CoRR, vol. abs/1907.01669, 2019. [Online]. Available: http://arxiv.org/abs/1907.01669

[13] F. Ladhak, A. Gandhe, M. Dreyer, L. Mathias, A. Rastrow, and B. Hoffmeister, "Latticernn: Recurrent neural networks over lattices," in INTERSPEECH, 2016.

[14] G. Jagfeld and N. T. Vu, "Encoding word confusion networks with recurrent neural networks for dialog state tracking," in Proceed ings of the Workshop on Speech-Centric Natural Language Processing. Copenhagen, Denmark: Association for Computational Linguistics, Sep. 2017, pp. 10-17.

[15] R. Masumura, Y. Ijima, T. Asami, H. Masataki, and R. Higashinaka, "Neural confnet classification: Fully neural network based spoken utterance classification using word confusion networks," 04 2018, pp. 6039-6043.
[16] J. Pennington, R. Socher, and C. D. Manning, "Glove: Global vectors for word representation," in In EMNLP, 2014

[17] K. Hashimoto, C. Xiong, Y. Tsuruoka, and R. Socher, "A joint many-task model: Growing a neural network for multiple NLP tasks," in Proceedings of the 2017 Conference on Empirical Methods in Natural Language Processing. Copenhagen, Denmark: Association for Computational Linguistics, Sep. 2017, pp. 1923-1933. [Online]. Available: https://www.aclweb.org/anthology/D17-1206 